Y. Yoshino

Nagoya Math. J.

Vol. 93 (1984), 39-60

\title{
SOME RESULTS ON THE VARIETY OF COMPLEXES
}

\author{
YUJI YOSHINO
}

\section{$\S 1$. Main theorems}

Let $R$ be a (commutative) Noetherian ring, and let $\left\{n_{0}, n_{1}, \cdots, n_{m}\right\}$ $\left\{k_{1}, k_{2}, \ldots, k_{m}\right\}$ be two sequences of integers satisfying $m>0, k_{i} \geqq 0(i=1$, $2, \cdots, m)$ and $n_{i} \geqq k_{i}+k_{i+1}(i=0,1,2, \cdots, m)$ with $k_{0}=k_{m+1}=0$. We consider the $m$-ple of matrices $\left(X^{(1)}, X^{(2)}, \ldots, X^{(m)}\right)$, where $X^{(s)}=\left(x_{i j}^{(s)}\right)$ is an $n_{s-1} \times n_{s}$ matrix of indeterminates over $R(s=1,2, \cdots, m)$. In this case we denote by $R\left[X^{(1)}, X^{(2)}, \cdots, X^{(m)}\right]$ a polynomial ring over $R$ with the indeterminates $\left\{x_{i_{s} s_{s}}^{(s)} \mid 1 \leqq s \leqq m, 1 \leqq i_{s} \leqq n_{s-1}, 1 \leqq j_{s} \leqq n_{s}\right\}$. Now we define an algebra $B_{R}\left(\begin{array}{c}n_{0}, n_{1}, \cdots, n_{m} \\ k_{1}, \cdots, k_{m}\end{array}\right)$ as follows,

$$
B_{R}\left(\begin{array}{c}
n_{0}, n_{1}, \cdots, n_{m} \\
k_{1}, \cdots, k_{m}
\end{array}\right):=R\left[X^{(1)}, X^{(2)}, \cdots, X^{(m)}\right] / I
$$

where $I$ is the ideal generated by all the elements of matrices $X^{(s-1)} X^{(s)}$ $(s=2,3, \cdots, m)$ and the minors of $X^{(s)}$ of size $k_{s}+1(s=1,2, \cdots, m)$.

These algebras first appeared in the following setting: In the affine space $\boldsymbol{A}_{R}^{N}=\oplus_{i=1}^{m} \operatorname{Hom}_{R}\left(\boldsymbol{A}_{R}^{n_{i}}, \boldsymbol{A}_{R}^{n_{i-1}}\right)$ if we define the subvariety $W\left(k_{1}, k_{2}, \cdots, k_{m}\right)$ of $A_{R}^{N}$ to be the closed subset consisting of $\left(f_{1}, f_{2}, \cdots, f_{m}\right)$ with $f_{i} \in$ $\operatorname{Hom}_{R}\left(A_{R}^{n_{i}}, A_{R}^{n_{i-1}}\right), f_{i} f_{i-1}=0(i=2,3, \cdots, m)$ and $\operatorname{rank}\left(f_{i}\right)=k_{i}(i=1,2, \cdots$, $m$ ), then the algebra $B_{R}\left(\begin{array}{c}n_{0}, n_{1}, \cdots, n_{m} \\ k_{1}, \cdots, k_{m}\end{array}\right)$ is nothing but the coordinate ring of $W\left(k_{1}, k_{2}, \cdots, k_{m}\right)$. (See [3; Theorem 1.7].)

These varieties are called the (Buchsbaum-Eisenbud) varieties of complexes. (Notice that for $m=1$, we obtain the determinantal varieties, whose properties have been considerably studied.)

Recently in their paper [3] DeConcini and Strickland have proved that $B_{R}\left(\begin{array}{c}n_{0}, n_{1}, \cdots, n_{m} \\ k_{1}, \cdots, k_{m}\end{array}\right)$ is normal iff $R$ is normal, and Cohen-Macaulay iff $R$ is Cohen-Macaulay, and their work encourages us to make our way

Received August 11, 1982. 
into the deeper study of these algebras.

In relation with their results, one of the purposes of the present paper is to determine the divisor class group of $B_{R}\left(\begin{array}{c}n_{0}, n_{1}, \cdots, n_{m} \\ k_{1}, \cdots, k_{m}\end{array}\right)$ in normal case and another is to get a necessary and sufficient condition for these algebras to be Gorenstein.

Our main theorems are the following.

Theorem 1.1. Let $R$ be a normal domain (and $\left\{n_{0}, n_{1}, \cdots, n_{m}\right\},\left\{k_{1} . k_{2}\right.$, $\left.\cdots, k_{m}\right\}$ as above). If we put $t_{i}=n_{i}-k_{i}-k_{i+1}(i=0,1, \cdots, m)$ with $k_{0}$ $=k_{m+1}=0$, then the divisor class group of $B_{R}\left(\begin{array}{c}n_{0}, n_{1}, \cdots, n_{m} \\ k_{1}, \cdots, k_{m}\end{array}\right)$ is given as follows;

$$
\mathrm{Cl}\left(B_{R}\left(\begin{array}{c}
n_{0}, n_{1}, \cdots, n_{m} \\
k_{1}, \cdots, k_{m}
\end{array}\right)\right)=\mathrm{Cl}(R) \oplus Z^{h}
$$

where $h=\#\left\{i \mid 0<k_{i}<n_{i}, t_{i-1}>0\right\}+\#\left\{i \mid 0<k_{i}<n_{i}, t_{i}=t_{i-1}=0\right\}$.

Theorem 1.2. Assume that $k_{i}>0(i=1,2, \cdots, m)$. If we denote $t_{i}$ $=n_{i}-k_{i}-k_{i+1}(i=0,1, \cdots, m)$, then $B_{R}\left(\begin{array}{c}n_{0}, n_{1}, \cdots, n_{m} \\ k_{1}, \cdots, k_{m}\end{array}\right)$ is a Gorenstein ring if and only if $R$ is Gorenstein and the integers $t_{0}, t_{1}, \cdots, t_{m}$ satisfy one of the following conditions;

(i ) $t_{0}=t_{1}=\cdots=t_{m}$,

(ii) $t_{0}=0, t_{1}=t_{2}=\cdots=t_{m}$,

(iii) $t_{m}=0, t_{0}=t_{1}=\cdots=t_{m-1}$,

(iv) $t_{0}=t_{m}=0, t_{1}=t_{2}=\cdots=t_{m-1}$.

Remark 1.3. The assumption that $k_{i}>0(i=1,2, \cdots, m)$ in Theorem. 1.2 is not so serious. In fact if $k_{s}=0$ for some $s$, then by the definition of those algebras we have an isomorphism;

$$
B_{R}\left(\begin{array}{c}
n_{0}, n_{1}, \cdots, n_{m} \\
k_{1}, \cdots, k_{m}
\end{array}\right) \simeq B_{R}\left(\begin{array}{c}
n_{0}, n_{1}, \cdots, n_{s-1} \\
k_{1}, \cdots, k_{s-1}
\end{array}\right) \otimes_{R} B_{R}\left(\begin{array}{c}
n_{s}, n_{s+1}, \cdots, n_{m} \\
k_{s+1}, \cdots, k_{m}
\end{array}\right) .
$$

So we can apply our theorem also in this case, because if $A$ and $B$ are finitely generated and faithfully flat $R$-algebras, then $A \otimes_{R} B$ is Gorenstein if and only if $A, B$ and $R$ are all Gorenstein. (Cf. [10; Theorem 2])

Remark 1.4. In the case $m=1$, our results are contained in Bruns [2] and Svanes [9] respectively. 
Whole of this paper is devoted to the proof of these theorems. Since our method is mainly based on the one developed by DeConcini and Strickland, we will often refer the reader to their paper [3].

The author wishes to thank Professor Kei-ichi Watanabe for his stimulative suggestion and helpful discussion on this topic.

\section{§2. Preliminaries}

Let $B_{R}\left(\begin{array}{c}n_{0}, n_{1}, \cdots, n_{m} \\ k_{1}, \cdots, k_{m}\end{array}\right)$ be as in the previous section. (We always assume that $k_{i} \geqq 0$ and $n_{i} \geqq k_{i}+k_{i+1}$ for any $i=0,1, \cdots, m$ with $k_{0}=$ $k_{m+1}=0$ and denote $t_{i}=n_{i}-k_{i}-k_{i+1}$ )

The symbol $\left[i_{1}, i_{2}, \cdots, i_{t} \mid j_{1}, j_{2}, \cdots, j_{t}\right]_{s}$ will denote the element of $B_{R}\left(\begin{array}{c}n_{0}, n_{1}, \cdots, n_{m} \\ k_{1}, \cdots, k_{m}\end{array}\right)$ which is given by the minor of the matrix $X^{(s)}$ whose rows are those of indices $i_{1}, i_{2}, \cdots, i_{t}$ and whose columns are those of indices $j_{1}, j_{2}, \cdots, j_{t}$. If $\left\{h_{1}, h_{2}, \cdots, h_{n_{s}-t}, j_{1}, \cdots, j_{t}\right\}=\left\{1,2, \cdots, n_{s}\right\}$, then $\left[i_{1}, i_{2}, \cdots, i_{t} \mid j_{1}, j_{2}, \cdots, j_{t}\right]_{s}$ can also be written as

$$
\sigma\left[i_{1}, \cdots, i_{t} \mid \hat{h}_{1}, \cdots, \hat{h}_{n_{s}-t}\right]_{s}
$$

where $\sigma$ is the sign of permutation $\left(h_{1}, \cdots, h_{n_{s}-t}, j_{1}, \cdots, j_{s}\right)$. (For more detail see $[3 ; \S 1])$.

The following lemma will be useful for us in proving inductively something about the property of these algebras.

Lemma 2.1. (See also [3; Lemma 2.10].) If we denote

$$
\begin{aligned}
a=\left[i_{1}, \cdots, i_{t} \mid j_{1}, \cdots, j_{t}\right]_{s} \quad\left(1 \leqq s \leqq m, 1 \leqq t \leqq k_{s},\right. \\
\left.1 \leqq i_{1}<i_{2}<\cdots<i_{t} \leqq n_{s-1}, 1 \leqq j_{1}<j_{2}<\cdots<j_{t} \leqq n_{s}\right),
\end{aligned}
$$

then there exists an isomorphism;

$$
B_{R}\left(\begin{array}{c}
n_{0}, n_{1}, \cdots, n_{m} \\
k_{1}, \cdots, k_{m}
\end{array}\right)\left[\frac{1}{a}\right] \simeq B_{\tilde{R}}\left(\begin{array}{c}
n_{0}, \cdots, n_{s-1}-t, n_{s}-t, n_{s+1}, \cdots, n_{m} \\
k_{1}, \cdots, k_{s}-t, k_{s+1}, \cdots, k_{m}
\end{array}\right)
$$

as $R$-algebras, where

$$
\tilde{R}=R\left[\left\{x_{i j}^{(s)} \mid i=i_{1} \cdots, i_{t} \text { or } j=j_{1}, \cdots, j_{t}\right\}, \frac{1}{a}\right] .
$$

Proof. To prove this lemma we have only to consider the case of $a=[1,2, \cdots, t \mid 1,2, \cdots, t]_{s}$. Then it is easy to see that there exist $P \in$ $G L\left(n_{s-1} ; \tilde{R}\right)$ and $Q \in G L\left(n_{s} ; \tilde{R}\right)$ such that 


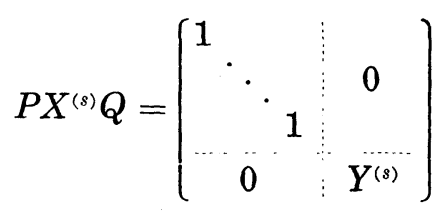

where $Y^{(s)}$ is an $\left(n_{s-1}-t\right) \times\left(n_{s}-t\right)$ matrix. Furthermore if we put

$$
Q^{-1} X^{(s+1)}=\left[\frac{*}{Y^{(s+1)}}\right]^{t} \text { and } X^{(s-1)} P^{-1}=[\underbrace{*}_{t} \mid Y^{(s-1)}]
$$

(we assume that $X^{(0)}=X^{(m+1)}=0$ ), and if $Y^{(i)}=X^{(i)}(i \neq s-1, s, s+1)$, then we can easily verify that $B_{R}\left(\begin{array}{c}n_{0}, n_{1}, \cdots, n_{m} \\ k_{1}, \cdots, k_{m}\end{array}\right)\left[\frac{1}{a}\right]$ is generated by those elements of $Y^{(i)}$ 's over $\tilde{R}$ and all their relations must be induced by those of $X^{(i)}$ 's. So we can consider this algebra to be the variety of complexes over $\tilde{R}$ with the generic matrices $\left(Y^{(1)}, \cdots, Y^{(m)}\right)$. We leave to the reader the detail of this proof.

Remark 2.2. If $a=[1, \cdots, t \mid 1, \cdots, t]_{s}$ is as in Lemma 2.1, then the matrices $P$ and $Q$ are given in the following form:

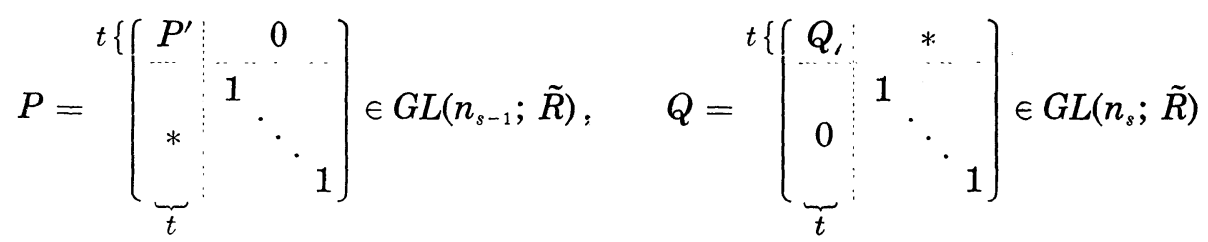

In this case let $\left[i_{1}, \cdots, i_{p} \mid j_{1}, \cdots, j_{p}\right]_{q}^{\sim}$ denote the element of

$$
B_{\tilde{R}}\left(\begin{array}{c}
n_{0}, n_{1}, \cdots, n_{s-1}-t, n_{s}-t, \cdots, n_{m} \\
k_{1}, \cdots, k_{s}-t, \cdots, k_{m}
\end{array}\right)
$$

which is given by the determinant of $\left[y_{i j}^{(q)}\right]_{\substack{i=i_{1}, \ldots, i_{p} \\ j=j_{1}, \ldots, j_{p}}}$ in the notation of the above proof.

Then by this remark it is not so difficult to see the following

LEMMA 2.3. Let $a=[1,2, \cdots, t \mid 1,2, \cdots, t]_{s}$ be as in Lemma 2.1 and $f$ be the natural map

$$
B_{R}\left(\begin{array}{c}
n_{0}, n_{1}, \cdots, n_{m} \\
k_{1}, \cdots, k_{m}
\end{array}\right) \longrightarrow B_{\tilde{R}}\left(\begin{array}{c}
n_{0}, \cdots, n_{s-1}-t, n_{s}-t, \cdots, n_{m} \\
k_{1}, \cdots, k_{s}-t, \cdots, k_{m}
\end{array}\right)
$$

which is given in Lemma 2.1 . 
(1) If $\left\{i_{1}, \cdots, i_{p}\right\} \subseteq\left\{t+1, t+2, \cdots, n_{s-1}\right\}$ and

$$
\begin{aligned}
& \left\{j_{1}, j_{2}, \cdots, j_{p}\right\} \subseteq\left\{t+1, t+2, \cdots, n_{s}\right\}, \text { then } \\
& \begin{array}{l}
f\left(\left[1,2, \cdots, t, i_{1}, \cdots, i_{p} \mid 1,2, \cdots, t, j_{1}, \cdots, j_{p}\right]_{s}\right) \\
\quad=u\left[i_{1}-t, i_{2}-t, \cdots, i_{p}-t \mid j_{1}-t, j_{2}-t, j_{p}-t\right]_{s}
\end{array}
\end{aligned}
$$

where $u$ is a unit in $\tilde{R}$.

(2) If $\left\{i_{1}, \cdots, i_{p}\right\} \subseteq\left\{t+1, t+2, \cdots, n_{s}\right\}$ and

$$
\left\{j_{1}, \cdots, j_{p}\right\} \subseteq\left\{1,2, \cdots, n_{s+1}\right\} \text {, then }
$$

$f\left(\left[i_{1}, \cdots, i_{p} \mid j_{1}, \cdots, j_{p}\right]_{s+1}\right)=\left[i_{1}-t, i_{2}-t, \cdots, i_{p}-t \mid j_{1}, \cdots, j_{p}\right]_{s+1}$.

(3) If $\left\{i_{1}, \cdots, i_{p}\right\} \subseteq\left\{1,2, \cdots, n_{s-2}\right\}$ and

$$
\begin{aligned}
& \left\{j_{1}, \cdots, j_{p}\right\} \subseteq\left\{t+1, t+2, \cdots, n_{s-1}\right\}, \text { then } \\
& f\left(\left[i_{1}, \cdots, i_{p} \mid j_{1}, \cdots, j_{p}\right]_{s-1}\right)=\left[i_{1}, \cdots, i_{p} \mid j_{1}-t, \cdots, j_{p}-t\right]_{s-1}^{\widetilde{N}}
\end{aligned}
$$

(4) If $q \neq s-1, s, s+1$, then

$$
f\left(\left[i_{1}, \cdots, i_{p} \mid j_{1}, \cdots, j_{p}\right]_{q}\right)=\left[i_{1}, \cdots, i_{p} \mid j_{1}, \cdots, j_{p}\right]_{q}^{\tilde{q}}
$$

In the ring $B_{R}\left(\begin{array}{c}n_{0}, n_{1}, \cdots, n_{m} \\ k_{1}, \cdots, k_{m}\end{array}\right)$ let $I^{(r)}\left(i_{1}, \cdots, i_{p} \mid j_{1}, \cdots, j_{q}\right)$ denote the ideal generated by the maximal minors of the matrix $\left[x_{i j}^{(r)}\right]_{\substack{i=i_{1}, \ldots, i_{p} \\ j=j_{1}, \ldots, j_{q}}}$ And in the same way we define $\tilde{I}^{(r)}\left(i_{1}, \cdots, i_{p} \mid j_{1}, \cdots, j_{q}\right)$ as the ideal of

$$
B_{\tilde{R}}\left(\begin{array}{c}
n_{0}, \cdots, n_{s-1}-t, n_{s}-t, \cdots, n_{m} \\
k_{1}, \cdots, k_{s}-t, \cdots, k_{m}
\end{array}\right)
$$

generated by the maximal minors of the matrix $\left[y_{i j}^{(r)}\right]_{\substack{i=i_{1}, \ldots, i_{p} \\ j=j_{1}, \ldots, j_{q}}}$ Then as a corollary of Lemma 2.3 we get

Corollary 2.4. Let $a=[1,2, \cdots, t \mid 1,2, \cdots, t]_{s}$ be as in Lemma 2.3, then

(1) $I^{(s)}\left(1,2, \cdots, t, i_{1}, \cdots, i_{p} \mid 1,2, \cdots, t, j_{1}, \cdots, j_{q}\right)\left[\frac{1}{a}\right]$

$$
=\tilde{I}^{(s)}\left(i_{1}-t, i_{2}-t, \cdots, i_{p}-t \mid j_{1}-t, j_{2}-t, \cdots, j_{q}-t\right)
$$

if $t<i_{u} \leqq n_{s-1}(u=1,2, \cdots, p)$ and $t<j_{v} \leqq n_{s}(v=1,2, \cdots, q)$.

(2) $I^{(s+1)}\left(1,2, \cdots, n_{s} \mid j_{1}, \cdots, j_{q}\right)\left[\frac{1}{a}\right]$

$$
=\tilde{I}^{(s+1)}\left(1,2, \cdots, n_{s}-t \mid j_{1}, \cdots, j_{q}\right) \text { if } q \leqq n_{s}-t .
$$

(3) $\quad I^{(s-1)}\left(i_{1}, \cdots, i_{p} \mid 1,2, \cdots, n_{s-1}\right)\left[\frac{1}{a}\right]$

$$
=\tilde{I}^{(s-1)}\left(i_{1}, \cdots, i_{p} \mid 1,2, \cdots, n_{s-1}-t\right) \text { if } p \leqq n_{s-1}-t .
$$


(4) $I^{(r)}\left(i_{1}, \cdots, i_{p} \mid j_{1}, \cdots, j_{q}\right)\left[\frac{1}{a}\right]=\tilde{I}^{(r)}\left(i_{1}, \cdots, i_{p} \mid j_{1}, \cdots, j_{q}\right)$ if $r \neq s-1, s, s+1$.

FACT 2.5. Let $H$ be the set consisting of all the determinants. We partially order $H$ by the following; When $x=\left[i_{1}, \cdots, i_{t} \mid j_{1}, \cdots, j_{t}\right]_{s}$ and $x^{\prime}=\left[i_{1}^{\prime}, \cdots, i_{t^{\prime}}^{\prime} \mid j_{1}^{\prime}, \cdots, j_{t^{\prime}}^{\prime}\right]_{s^{\prime}}, x$ and $x^{\prime}$ are incomparable if $s \neq s^{\prime}$, while if $s=s^{\prime}$ then $x \leqq x^{\prime}$ if $t \geqq t^{\prime}$ and $i_{u} \leqq i_{u}^{\prime}, j_{u} \leqq j_{u}^{\prime}$ for $u=1,2, \cdots, t^{\prime}$. The product $x x^{\prime}\left(s^{\prime} \geqq s\right)$ is said to be standard if one of the following conditions holds;

(1) $s^{\prime}>s+1$

(2) $s^{\prime}=s$ and $x$ and $x^{\prime}$ are comparable in the partial order on $H$.

(3) $s^{\prime}=s+1, n_{s}-t \geqq t^{\prime}$ and writing $u_{1}<u_{2}<\cdots<u_{n_{s}-t}$ for the complement of $\left\{j_{1}, \cdots, j_{t}\right\}$ in $\left\{1,2, \cdots, n_{s}\right\}$, we have

$$
u_{n_{s}-t-v+1} \geqq j_{t^{\prime}-v+1} \quad \text { for } v=1,2, \cdots, t^{\prime} .
$$

(If we symbolically write $x=[I \mid J]_{s}, x^{\prime}=\left[I^{\prime} \mid J^{\prime}\right]_{s+1}$ and if we define a permutation $\pi_{s}$ on $\left\{1,2, \cdots, n_{s}\right\}$ as

$$
\pi_{s}(u)=n_{s}-u+1 \quad\left(1 \leqq u \leqq n_{s}\right),
$$

then this condition could be re-written as $\pi_{s}(\hat{J}) \leqq \pi_{s}\left(I^{\prime}\right)$.)

Now we define an arbitrary product $x_{1} x_{2} \cdots x_{n}$ of minors to be standard if each product $x_{i} x_{j}$ is standard in a suitable order.

Then the theorem of DeConcini and Strickland says that the algebra $B_{R}\left(\begin{array}{c}n_{0}, n_{1}, \cdots, n_{m} \\ k_{1}, \cdots, k_{m}\end{array}\right)$ is a Hodge algebra over $R$ generated by $H$. (Cf. [3] and $[5 ; \S 16])$

Remark. Our notion of standardness does not coincide with that of [3]. In fact the condition (3) above corresponds to the standardness given in [3] by considering the product matrices

$$
X^{(s)}\left[{ }_{1} \cdot{ }^{1}\right], \quad\left[{ }_{1}^{\cdot}\right]^{1} X^{(s+1)}
$$

instead of $X^{(s)}$ and $X^{(s+1)}$. This is necessary if one wishes to make $B_{R}\left(\begin{array}{c}n_{0}, n_{1}, \cdots, n_{m} \\ k_{1}, \cdots, k_{m}\end{array}\right)$ a Hodge algebra defined in [5].

LEMmA 2.6. Suppose that $R$ is a reduced ring and $k_{1} \geqq 1$. If we denote 
$d=\left[1,2, \cdots, k_{1} \mid 1,2, \cdots, k_{1}\right]_{1}$ and $\mathfrak{p}=I^{(1)}\left(1,2, \cdots, k_{1} \mid 1,2, \cdots, n_{1}\right)$, then the ideals $(d)$ and $p$ are radical ideals.

Moreover if $m \geqq 2, k \geqq 1$ and if we denote $w=\left[1,2, \cdots, k_{m} \mid 1,2, \cdots, k_{m}\right]_{m}$ then $w$ is a non zero divisor on $B / p$ and $B /(d)$, where

$$
B=B_{R}\left(\begin{array}{c}
n_{0}, n_{1}, \cdots, n_{m} \\
k_{1}, \cdots, k_{m}
\end{array}\right) \text {. }
$$

Proof. Recall that a subset $I$ of $H$ is called a poset ideal if $I \ni a \geqq b$ $\in H$ implies $b \in I$. In our case $\{d\}$ and the system of generators

$$
\left\{\left[1,2, \cdots, k_{1} \mid i_{1}, \cdots, i_{k_{1}}\right]_{1} \mid 1 \leqq i_{1}<i_{2}<\cdots<i_{k_{1}} \leqq n_{1}\right\}
$$

of $\mathfrak{p}$ are in fact poset ideals of $H$. (Notice that $d$ is a minimal element in $H$.) Thus by Proposition 1.2 of [5] we have that both $B /(d)$ and $B / p$ are also Hodge algebras over $R$. In particular they are reduced.

Next assume that $w\left(\sum_{i} a_{i} x_{i}\right) \in \mathfrak{p}$ (resp. $\in(d)$ ) where each $x_{i}$ is a standard monomial and $a_{i} \in R$. By the straightening law we can write $w x_{i}=$ $\sum_{j} b_{i j} y_{i j}\left(y_{i j}\right.$ is a standard monomial and $\left.b_{i j} \in R\right)$. In this expression each monomial $y_{i j}$ contains $w$, for $w$ is minimal in the order on $H$. Since $\sum_{i, j} a_{i} b_{i j} y_{i j} \in \mathfrak{p}$ (resp. $\in(d)$ ) and since this expression is unique, each $y_{i j}$ also contains one of $\left[1,2, \cdots, k_{1} \mid i_{1}, \cdots, i_{k_{1}}\right]_{1}\left(1 \leqq i_{1}<\cdots<i_{k_{1}} \leqq n_{1}\right)$ (resp. $\left.d\right)$.

Thus we have $w\left(\sum a_{i} x_{i}\right) \in w$ p. (resp. $\in(w d)$ ). Because $w$ is a non zero divisor on $B$, we conclude that $\sum_{i} a_{i} x_{i} \in \mathfrak{p}$ (resp. $\in(d)$ ). Hence $w$ is a non zero divisor on $B / \mathfrak{p}$ (resp. $B /(d)$ ).

Now we shall prove the main proposition of this section.

Proposition 2.7. Assume that $R$ is an integral domain and $k_{1} \geqq 1$. If we put $\mathfrak{p}=I^{(1)}\left(1,2, \cdots, k_{1} \mid 1,2, \cdots, n_{1}\right)$ as in Lemma 2.6, then $\mathfrak{p}$ is a prime ideal and

$$
\operatorname{ht}(\mathfrak{p})= \begin{cases}1 & \left(k_{1}<n_{0}\right) \\ n_{1}-k_{1}-k_{2}+1 & \left(k_{1}=n_{0}\right) .\end{cases}
$$

Proof. We prove the proposition by the induction on $m$. If $m=1$, then this algebra $B:=B_{R}\left(\begin{array}{c}n_{0}, n_{1} \\ k_{1}\end{array}\right)$ is the determinantal variety and so the result has been known by [6; Theorem 1].

So assume that $m \geqq 2$. If we denote $w=\left[1,2, \cdots, k_{m} \mid 1,2, \cdots, k_{m}\right]_{m}$, then by Lemma 2.6 we have only to prove that $\mathfrak{p} B_{w}$ is prime and its height is given as in the proposition. 
On the other hand $B_{w}$ is isomorphic to $\widetilde{B}:=B_{\tilde{R}}\left(\begin{array}{c}n_{0}, \cdots, n_{m-2}, n_{m-1}-k_{m} \\ k_{1}, \cdots, k_{m-1}\end{array}\right)$ with a certain integral domain $\widetilde{R}$ and by this isomorphism $\mathfrak{p} B_{w}$ corresponds to the ideal $\tilde{p}$ which is defined in $\widetilde{B}$ as in the same way as $\mathfrak{p}$. (See Corollary 2.4.)

By the induction hypothesis $\tilde{\mathfrak{p}}$ is in fact a prime ideal and

$$
\operatorname{ht}(\tilde{\mathfrak{p}})= \begin{cases}1 & \left(k_{1}<n_{0}\right) \\ n_{1}-k_{1}-k_{2}+1 & \left(k_{1}=n_{0}, m \geqq 3\right) \\ \left(n_{1}-k_{2}\right)-k_{1}+1 & \left(k_{1}=n_{0}, m=2\right) .\end{cases}
$$

This is what we wanted.

Remark 2.8. $I:=I^{(1)}\left(1,2, \cdots, n_{0} \mid 1,2, \cdots, k_{1}\right)$ may not be a prime ideal. In fact if we consider

$$
B:=B_{R}\left(\begin{array}{c}
1,2,2 \\
1,1
\end{array}\right)=R[u, v, x, y, z, w] /(u x+v z, u y+v w, x w-y z),
$$

then $I=(u), \mathfrak{p}=(u, v)$ and so

$$
B / I \simeq R[v, x, y, z, w] /(v z, v w, x w-y z)
$$

which is not an integral domain, while

$$
B / \mathfrak{p} \simeq R[x, y, z, w] /(x w-y z)
$$

is an integral domain whenever $R$ is a domain.

\section{§3. Proof of Theorem 1.1.}

In order to prove Theorem 1.1 we need some lemmas on the decomposition of a certain principal ideal, which will be stated in (3.2).

In the following we always denote $B:=B_{R}\left(\begin{array}{c}n_{0}, n_{1}, \cdots, n_{m} \\ k_{1}, \cdots, k_{m}\end{array}\right)$.

Lemma 3.1. Assume that $R$ is an integral domain and $1 \leqq k_{1}<n_{1}$. If we denote $\mathfrak{p}=I^{(1)}\left(1,2, \cdots, k_{1} \mid 1,2, \cdots, n_{1}\right)$

$$
d=\left[1,2, \cdots, k_{1} \mid 1,2, \cdots, k_{1}\right]_{1}
$$

and $\quad e=\left[1,2, \cdots, k_{1} \mid 1,2, \cdots, k_{1}-1, k_{1}+1\right]_{1}$,

then we have $(d)=\mathfrak{p} \cap\left(d B_{e} \cap B\right)$.

Proof. It is enough to see that for an element $x$ of $\mathfrak{p}, e x \in(d)$ implies $x \in(d)$. 
Let us describe $x$ as a linear combination of standard monomials as follows;

$$
x=\sum_{i} a_{i} x_{i} \quad\left(a_{i} \in R\right)
$$

where each standard monomial $x_{i}$ contains one of $\left[1,2, \cdots, k_{1} \mid i_{1}, \cdots, i_{k_{1}}\right]_{1}$ $\left(1 \leqq i_{1}<\cdots<i_{k_{1}} \leqq n_{1}\right)$, since $x \in \mathfrak{p}$.

Assume that $x_{i}$ does not contain $d$. Then by the definition of standardness we can easily check that $e x_{i}$ is also a standard monomial. Since $e x \in(d)$ and since $d$ is a minimal element of $H$, the straightening law shows that the monomial $e x_{i}$ contains $d$. It also means that $x_{i}$ contains $d$, which is a contradiction. Thus $d$ is included in each monomial $x_{i}$, so $x \in(d)$.

Proposition 3.2. Assume that $R$ is an integral domain, $k_{1}>0$ and $d:=\left[1,2, \cdots, k_{1} \mid 1,2, \cdots, k_{1}\right]_{1}$.

(1) If $k_{1}<\min \left\{n_{0}, n_{1}\right\}$ or $k_{1}=n_{0}=n_{1}-k_{2}<n_{1}$ then we have

$$
(d)=\mathfrak{p} \cap \mathfrak{q}
$$

where $\mathfrak{p}=I^{(1)}\left(1,2, \cdots, k_{1} \mid 1,2, \cdots, n_{1}\right)$, which is a prime ideal of height one as shown in Proposition 2.7 and $\mathfrak{q}$ denotes another prime ideal of height one.

(2) If $k_{1}=n_{1}$ or $k_{1}=n_{0}<n_{1}-k_{2}$, then the ideal $(d)$ is prime itself.

Proof. If $k_{1}=n_{1}$, then $(d)=\mathfrak{k}$, so it is prime by Proposition 2.7.

Assume that $k_{1}<n_{1}$. In this case let us denote

$$
e=\left[1,2, \cdots, k_{1} \mid 1,2, \cdots, k_{1}-1, k_{1}+1\right]_{1}
$$

as in Lemma 3.1. First we claim that $d B_{e}$ is a proper prime ideal.

In fact we have already known that $B_{e} \simeq B_{\tilde{R}}\left(\begin{array}{c}n_{1}-k_{1}, n_{n}, \cdots, n_{m} \\ k_{2}, \cdots, k_{m}\end{array}\right)$ with $\tilde{R}=R\left[\left\{x_{i j}^{(1)} \mid i=1,2, \cdots, k_{1}\right.\right.$ or $\left.\left.j=1,2, \cdots, k_{1}-1, k_{1}+1\right\}, 1 / e\right]$ and by this isomorphism $d$ corresponds to the element $\tilde{d}:=\operatorname{det}\left[x_{i j}^{(1)}\right]_{\substack{i=1,2, \ldots, k_{1} \\ j=1,2, \ldots, k_{1}}}$ of $R$. It is well known that $\tilde{d}$ is a prime element of $\tilde{R}$. (See [6; Theorem 1].) Hence $B_{e} / d B_{e} \simeq B_{\tilde{R} / d \tilde{R}}\left(\begin{array}{c}n_{1}-k_{1}, n_{2}, \cdots, n_{m} \\ k_{2}, \cdots, k_{m}\end{array}\right)$ which is in fact an integral domain, and so we conclude that $d B_{e}$ is prime as we wanted.

Thus if we denote $\mathfrak{q}=d B_{e} \cap B$, then by Lemma 3.1 we get the decomposition of $(d)$ into prime ideals; 
(Remark that clearly $\mathfrak{p} \not \subset \mathfrak{q}$.)

In the first case of proposition, the height of $p$ is one as shown in Proposition 2.7. So $(*)$ gives the irredundant decomposition of $(d)$.

On the other hand if $k_{1}=n_{0}<n_{1}-k_{2}$, then $\mathrm{ht}(\mathfrak{p}) \geqq 2$ by Proposition 2.7, hence $q$ must be contained in $p$ since $(d)$ is a radical ideal. Q.E.D.

Proof of Theorem 1.1. Assume that $R$ is a normal domain as in the assumption of Theorem 1.1 .

To prove the theorem it will be sufficient to see the following claim;

$$
\mathrm{Cl}\left(B_{R}\left(\begin{array}{c}
n_{0}, n_{1}, \cdots, n_{m} \\
k_{1}, \cdots, k_{m}
\end{array}\right)\right) \simeq Z^{e} \oplus \mathrm{Cl}\left(B_{\tilde{R}}\left(\begin{array}{c}
n_{1}-k_{1}, n_{2}, \cdots, n_{m} \\
k_{2}, \cdots, k_{m}
\end{array}\right)\right)
$$

where

$$
e= \begin{cases}1 & \text { (if } \left.0<k_{1}<\min \left\{n_{0}, n_{1}\right\} \text { or } 0<k_{1}=n_{0}=n_{1}-k_{2}<n_{1}\right) \\ 0 & \text { (otherwise) }\end{cases}
$$

where $\tilde{R}$ is a certain normal domain which is a faithfully flat $R$-algebra and has the same divisor class group as $R$.

If the claim (3.3) is verified, then we can prove Theorem 1.1 by the induction on $m$. In fact if $m=1$, then by the claim we have

$$
\mathrm{Cl}\left(B_{R}\left(\begin{array}{c}
n_{0}, n_{1} \\
k_{1}
\end{array}\right)\right) \simeq Z^{e} \oplus \mathrm{Cl}(R)
$$

where

$$
e= \begin{cases}1 & \text { if } 0<k_{1}<\min \left\{n_{0}, n_{1}\right\} \\ 0 & \text { otherwise }\end{cases}
$$

that is nothing but Theorem 1.1 in case $m=1$.

If $m>1$ and if we put

$$
\begin{aligned}
h\left(\begin{array}{c}
n_{0}, n_{1}, \cdots, n_{m} \\
k_{1}, \cdots, k_{m}
\end{array}\right)= & \#\left\{i \mid 0<k_{i}<n_{i}, t_{i-1}>0\right\} \\
& +\#\left\{i \mid 0<k_{i}<n_{i}, t_{i-1}=t_{i}=0\right\}
\end{aligned}
$$

(recall that $t_{i}=n_{i}-k_{i}-k_{i+1}$ ), then by (3.3) and by the induction hypothesis we get

$$
\mathrm{Cl}\left(B_{R}\left(\begin{array}{c}
n_{0}, n_{1}, \cdots, n_{m} \\
k_{1}, \cdots, k_{m}
\end{array}\right)\right) \simeq Z^{n} \oplus \mathrm{Cl}(R)
$$


where $h=e+h\left(\begin{array}{c}n_{1}-k_{1}, n_{2}, \cdots, n_{m} \\ k_{2}, \cdots, k_{m}\end{array}\right)$. Here it is not so difficult to see that $h=h\left(\begin{array}{c}n_{0}, n_{1}, \cdots, n_{m} \\ k_{1}, \cdots, k_{m}\end{array}\right)$.

Now we proceed to the proof of (3.3).

Our proof will be divided into the following three cases;

(1) $k_{1}=0$,

(2) $k_{1}=n_{1}$ or $k_{1}=n_{0}<n_{1}-k_{2}$,

(3) $0<k_{1}<\min \left\{n_{0}, n_{1}\right\}$ or $0<k_{1}=n_{0}=n_{1}-k_{2}<n_{1}$.

(Observe that they exhaust all the possible cases.)

In case (1) it is clear that $B_{R}\left(\begin{array}{c}n_{0}, n_{1}, \cdots, n_{m} \\ k_{1}, \cdots, k_{m}\end{array}\right)=B_{R}\left(\begin{array}{c}n_{1}, n_{2}, \cdots, n_{m} \\ k_{2}, \cdots, k_{m}\end{array}\right)$. Thus there is nothing to prove.

In case (2) the element $d=\left[1,2, \cdots, k_{1} \mid 1,2, \cdots, k_{1}\right]_{1}$ is a prime element by Proposition 3.2. Therefore the divisor class group does not change after localizing by $d$. On the other hand there is an isomorphism

$$
B_{R}\left(\begin{array}{c}
n_{0}, n_{1}, \cdots, n_{m} \\
k_{1}, \cdots, k_{m}
\end{array}\right)\left[\frac{1}{d}\right] \simeq B_{\tilde{R}}\left(\begin{array}{c}
n_{1}-k_{1}, n_{2}, \cdots, n_{m} \\
k_{2}, \cdots, k_{m}
\end{array}\right)
$$

as we have shown in Lemma 2.1. Since

$$
\tilde{R}=R\left[\left\{[i \mid j]_{1} \mid 1 \leqq i \leqq k_{1}, 1 \leqq j \leqq k_{1}\right\}, 1 / d\right]
$$

and since $d$ is a prime element of $R\left[\left\{[i \mid j]_{1} \mid 1 \leqq i \leqq k_{1}, 1 \leqq j \leqq k_{1}\right\}\right]$ (see [6; Theorem 1]), we have an isomorphism $\mathrm{Cl}(R) \simeq \mathrm{Cl}(\tilde{R})$. Hence we get the claim in this case.

In case (3) the ideal $(d)$ is decomposed into the intersection of two prime ideals;

$$
(d)=\mathfrak{p} \cap \mathfrak{q}
$$

as shown in Proposition 3.2. If $D(A)$ denotes the free abelian group generated by height-one prime ideals in a normal domain $A$, and $F(A)$ the subgroup of $D(A)$ consisting of principal divisors, then we have the following commutative diagram induced by the natural map $B \rightarrow B_{d}$, where $B=B_{R}\left(\begin{array}{c}n_{0}, n_{1}, \cdots, n_{m} \\ k_{1}, \cdots, k_{m}\end{array}\right)$.

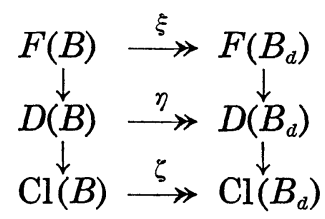


Immediately it can be known that

$$
\begin{aligned}
& \operatorname{Ker}(\eta)=Z \operatorname{div}(\mathfrak{p})+Z \operatorname{div}(\mathfrak{q}) \\
& \operatorname{Ker}(\xi)=Z \operatorname{div}(d)
\end{aligned}
$$

where "div" expresses the class in $D(B)$. (Notice that $F(B) \simeq Q(B)-$ $\{0\} / U(B)$ where $Q(B)$ is the quotient field of $B$ and $U(B)$ the group of units in $B$. See [1; Chapter 7].) Furthermore by the above decomposition of $(d)$ it is clear that $\operatorname{div}(d)=\operatorname{div}(\mathfrak{p})+\operatorname{div}(\mathfrak{q})$ in $D(B)$. Hence we conclude that the kernel of $\zeta$ is generated by $\mathrm{cl}(\mathfrak{p})$ and it is $Z$-free. Anyway we get the following exact sequence of abelian groups;

$$
\begin{aligned}
&(*) \quad 0 \longrightarrow Z \longrightarrow \operatorname{Cl}(B) \stackrel{\zeta}{\longrightarrow} \mathrm{Cl}(\tilde{B}) \longrightarrow 0 \\
& 1 \longmapsto \operatorname{cl}(\mathfrak{p})
\end{aligned}
$$

where $B:=B_{d} \simeq B_{\tilde{R}}\left(\begin{array}{c}n_{1}-k_{1}, n_{2}, \cdots, n_{m} \\ k_{2}, \cdots, k_{m}\end{array}\right)$ as in Lemma 2.1.

It remains only to prove that this sequence $(*)$ is split. We shall prove this by the induction on $m$.

Since we may assume that $\tilde{B}$ also satisfies our claim (3.3), $\mathrm{Cl}(\tilde{B})$ can be expressed by the direct sum of $\mathrm{Cl}(R)$ and $Z^{\tilde{h}}$ for some $\tilde{h} \geqq 0$, as we have shown above. On the other hand by the diagram of algebras;<smiles>[R][R]1[R][B]1</smiles>

we get the following commutative diagram of abelian groups;

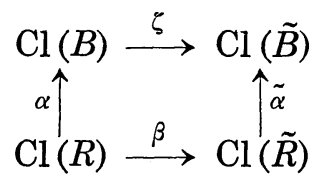

where $\beta$ is an isomorphism as in the proof of case (2).

If $m=1, \tilde{\alpha}$ is also an isomorphism and so $\zeta$ has the right inverse $\alpha \beta^{-1} \tilde{\alpha}^{-1}$ as wanted.

Assume that $m>1$. Then by the induction hypothesis $\tilde{\alpha}$ has the left inverse $\delta$ and the cokernel of $\tilde{\alpha}$ is isomorphic to $Z^{\tilde{h}}$. So there exists a map $\gamma: Z^{\tilde{n}} \rightarrow \mathrm{Cl}(B)$ such that $\pi \zeta \gamma$ gives the identity map on $Z^{\tilde{n}}$ where $\pi$ denotes the natural projection from $\mathrm{Cl}(\tilde{B})$ to $\operatorname{Coker}(\tilde{\alpha}) \simeq Z^{h}$. Thus we can conclude that $\alpha \beta^{-1} \delta \oplus \gamma$ is the right inverse of $\zeta$. 
This completes the proof of (3.3) and so Theorem 1.1 .

Remark 3.4. There exists an automorphism of $B:=B_{R}\left(\begin{array}{c}n_{0}, n_{1}, \cdots, n_{m} \\ k_{1}, \cdots, k_{m}\end{array}\right)$ which permutes some columns of the matrix $X^{(1)}$ and leaves the ideal $p$ stable. From this fact we see that even if $d$ denotes $\left[1,2, \cdots, k_{1} \mid i_{1}, \cdots, i_{k_{1}}\right]_{1}$ for arbitrary $i_{1}<\cdots<i_{k_{1}} \leqq n_{1}$ instead of $\left[1,2, \cdots, k_{1} \mid 1,2, \cdots, k_{1}\right]_{1}$, then the natural map from $\mathrm{Cl}(B)$ to $\mathrm{Cl}\left(B_{d}\right)$ is an isomorphism in case (2) and the sequence $(*)$ is also split exact in case (3).

Carefully pursuing the proof of the theorem, we shall have the following corollary which will be used in Sections 4 and 5 to prove Theorem 1.2 .

Corollary 3.5. Let us denote $B:=B_{R}\left(\begin{array}{c}n_{0}, n_{1}, \cdots, n_{m} \\ k_{1}, \cdots, k_{m}\end{array}\right)$ with a normal domain $R$ and $x:=[1 \mid 1]_{s}$ for some $s$. If one of the following conditions holds;

(i) $k_{s} \geqq 2$,

(ii) $s=1, k_{1}=k_{2}=n_{0}=1$ and $n_{1}>2$,

(iii) $s=m, k_{m}=k_{m-1}=n_{m}=1$ and $n_{m-1}>2$,

then the group homomorphism $\mathrm{Cl}(B) \rightarrow \mathrm{Cl}\left(B_{x}\right)$ induced by the natural map of $B$ to $B_{x}$ is an isomorphism.

Proof. In case (ii) or (iii) the element $x$ is prime by Proposition 3.2 and so the result will be obtained by [1; Chapter $7 \S 1$ Proposition 17].

In case (i) we prove this by the induction on $m$. It is also divided into the following three cases as in the proof of (3.3):

(1) $k_{1}=0$,

(2) $k_{1}=n_{1}>0$ or $0<k_{1}=n_{0}<n_{1}-k_{2}$,

(3) $0<k_{1}<\min \left\{n_{0}, n_{1}\right\}$ or $0<k_{1}=n_{0}=n_{1}-k_{2}<n_{1}$.

In case (1) this obviously reduces to the case with less $m$ since $B \simeq B_{R}\left(\begin{array}{c}n_{1}, n_{2}, \cdots, n_{m} \\ k_{2}, \cdots, k_{m}\end{array}\right)$.

In case (2) let us denote

$$
d= \begin{cases}{\left[1,2, \cdots, k_{1} \mid 1,2, \cdots, k_{1}\right]_{1}} & \text { if } s \neq 2, \\ {\left[1,2, \cdots, k_{1} \mid n_{1}-k_{1}+1, \cdots, n_{1}\right]_{1}} & \text { if } s=2,\end{cases}
$$

and 


$$
\tilde{d}= \begin{cases}{\left[1,2, \cdots, k_{1}-1 \mid 1,2, \cdots, k_{1}-1\right]_{1}^{\sim}} & \text { if } s=1, \\ {\left[1,2, \cdots, k_{1} \mid n_{1}-k_{1}, \cdots, n_{1}-1\right]_{1}^{\sim}} & \text { if } s=2, \\ {\left[1,2, \cdots, k_{1} \mid 1,2, \cdots, k_{1}\right]_{1}} & \text { if } s \geqq 3 .\end{cases}
$$

Then by Lemma 2.3 we know that $d$ corresponds to $\tilde{d}$ under the natural map from $B$ to $B_{x}$. (Notice that in case $s=2, n_{1}-k_{1} \geqq k_{2} \geqq 2$.) Applying Remark 3.4 to $B$ and $B_{x}$, we get the commutative diagram;

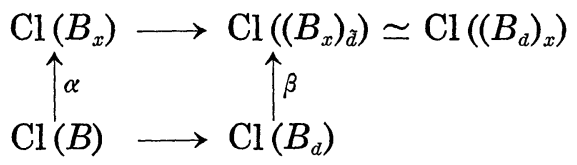

where the horizontal arrows are isomorphisms. On the other hand the induction hypothesis shows that $\beta$ is also an isomorphism. Thus $\alpha$ is an isomorphism as we wanted.

In case (3) let $d$ and $\tilde{d}$ be as above and let $\tilde{\mathfrak{p}}$ denote the ideal;

or

$$
\begin{array}{ll}
\tilde{I}^{(1)}\left(1,2, \cdots, k_{1}-1 \mid 1,2, \cdots, n_{1}-1\right) & \text { if } s=1, \\
\tilde{I}^{(1)}\left(1,2, \cdots, k_{1} \mid 1,2, \cdots, n_{1}-1\right) & \text { if } s=2, \\
\tilde{I}^{(1)}\left(1,2, \cdots, k_{1} \mid 1,2, \cdots, n_{1}\right) & \text { if } s \geqq 3 .
\end{array}
$$

Then $d$ (resp. $\mathfrak{p}$ ) corresponds to $\tilde{d}$ (resp. $\tilde{\mathfrak{p}}$ ) as we have shown in Lemma 2.3 and Corollary 2.4. Thus we obtain the following commutative diagram with exact rows.

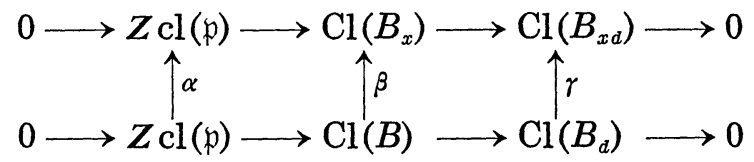

(Notice that $\alpha$ is an isomorphism.) On the other hand by the hypothesis of induction we can easily see that $\gamma$ is an isomorphism. So it is concluded that $\beta$ is also an isomorphism.

Q.E.D.

\section{§4. Reduction}

In this section we shall show that it is sufficient to prove the following proposition in order to get Theorem 1.2.

Proposition 4.1. Assume that $R$ is a field. Then $B_{R}\left(\begin{array}{c}n_{0}, n_{1}, \cdots, n_{m} \\ 1, \cdots, 1\end{array}\right)$ is Gorenstein if and only if one of the following four conditions holds:

(1) $n_{0}+1=n_{1}=n_{2}=\cdots=n_{m-1}=n_{m}+1$, 
(2) $n_{0}=1, n_{1}=n_{2}=\cdots=n_{m-1}=n_{m}+1$,

(3) $n_{m}=1, n_{0}+1=n_{1}=n_{2}=\cdots=n_{m-1}$,

(4) $n_{0}=n_{m}=1, n_{1}=n_{2}=\cdots=n_{m-1}$.

The proof of this proposition will be done in the next section, and for a while we assume that this holds actually.

For the purpose of reducing Theorem 1.2 to this proposition we need some lemmas, the following one of which is rather well known.

LEMMA 4.2. (1) $B_{R}\left(\begin{array}{c}n_{0}, n_{1}, \cdots, n_{m} \\ k_{1}, \cdots, k_{m}\end{array}\right)$ is Gorenstein if and only if $R$ and $B_{k(\mathfrak{p})}\left(\begin{array}{c}n_{0}, n_{1}, \cdots, n_{m} \\ k_{1}, \cdots, k_{m}\end{array}\right)$ are Gorenstein for any prime ideal $\mathfrak{p}$ of $R$ where $k(\mathfrak{p})$ $=R_{\mathrm{p}} / \mathfrak{p} R_{\mathrm{p}}$.

(2) Let $R \rightarrow S$ be a faithfully flat extention of Noetherian rings with Gorenstein fibres. Then $B_{R}\left(\begin{array}{c}n_{0}, n_{1}, \cdots, n_{m} \\ k_{1}, \cdots, k_{m}\end{array}\right)$ is Gorenstein if and only if $B_{S}\left(\begin{array}{c}n_{0}, n_{1}, \cdots, n_{m} \\ k_{1}, \cdots, k_{m}\end{array}\right)$ is Gorenstein.

Proof. (1) Since the natural map $R \rightarrow B_{R}\left(\begin{array}{c}n_{0}, n_{1}, \cdots, n_{m} \\ k_{1}, \cdots, k_{m}\end{array}\right)$ is faithfully flat, the result is obtained from [10; Theorem 1].

(2) By the assumption we see that the natural map from

$$
B_{R}\left(\begin{array}{c}
n_{0}, n_{1}, \cdots, n_{m} \\
k_{1}, \cdots, k_{m}
\end{array}\right) \text { to } B_{S}\left(\begin{array}{c}
n_{0}, n_{1}, \cdots, n_{m} \\
k_{1}, \cdots, k_{m}
\end{array}\right)
$$

is faithfully flat. Thus if $B_{S}\left(\begin{array}{c}n_{0}, n_{1}, \cdots, n_{m} \\ k_{1}, \cdots, k_{m}\end{array}\right)$ is Gorenstein, then by [10; Theorem 1] we see that $B_{R}\left(\begin{array}{c}n_{0}, n_{1}, \cdots, n_{m} \\ k_{1}, \cdots, k_{m}\end{array}\right)$ is also Gorenstein. Conversely assume that $B_{R}\left(\begin{array}{c}n_{0}, n_{1}, \cdots, n_{m} \\ k_{1}, \cdots, k_{m}\end{array}\right)$ is Gorenstein. Then $R$ is Gorenstein and so is $S$. (Use (1) and [10; Theorem 1].) Hence the Gorensteinness of

$$
B_{S}\left(\begin{array}{c}
n_{0}, n_{1}, \cdots, n_{m} \\
k_{1}, \cdots, k_{m}
\end{array}\right) \simeq B_{R}\left(\begin{array}{c}
n_{0}, n_{1}, \cdots, n_{m} \\
k_{1}, \cdots, k_{m}
\end{array}\right) \otimes_{R} S .
$$

follows from [10; Theorem 2].

LEMMA 4.3. Let us denote $B=B_{R}\left(\begin{array}{c}n_{0}, n_{1}, \cdots, n_{m} \\ k_{1}, \cdots, k_{m}\end{array}\right)$ and $x=[1 \mid 1]_{s}$ for some s. If one of the following conditions holds;

(i) $k_{s} \geqq 2$, 
(ii) $s=1, k_{1}=k_{2}=n_{0}=1$ and $n_{1}>2$,

(iii) $s=m, k_{m}=k_{m-1}=n_{m}=1$ and $n_{m-1}>2$,

then $B$ is Gorenstein if and only if $B_{x}$ is Gorenstein.

Proof. By the above lemma we may assume that $R$ is a field, and so we can consider the canonical module $K_{B}$ of the graded ring $B$. (For the definition of $K_{B}$ see [7; Chapter 2].) Remark that $K_{B}$ is isomorphic to a certain divisorial ideal of $B$ since $B$ is a Cohen-Macaulay normal domain. (Cf. [8; Korollar 6.7]) Therefore $K_{B}$ defines the divisor class $\operatorname{cl}\left(K_{B}\right)$ in $\mathrm{Cl}(B)$ and thus $B$ is Gorenstein if and only if $\operatorname{cl}\left(K_{B}\right)=0$. (See [8; Satz 5.9].)

If we denote $B^{\prime}=B_{R}\left(\begin{array}{c}n_{0}, n_{1}, \cdots, n_{s-1}-1, n_{s}-1, \cdots, n_{m} \\ k_{1}, \cdots, k_{s}-1, \cdots, k_{m}\end{array}\right)$ and $\tilde{R}=$ $R\left[\left\{[i \mid j]_{s} \mid i=1\right.\right.$ or $\left.\left.j=1\right\}, 1 / x\right]$, then $B_{x} \simeq B^{\prime} \otimes_{R} \tilde{R}$ as we have shown in Lemma 2.1, and so we have the natural maps among the divisor class groups;

$$
\mathrm{Cl}(B) \stackrel{f}{\longrightarrow} \mathrm{Cl}\left(B_{x}\right) \simeq \mathrm{Cl}\left(B^{\prime} \otimes_{R} \tilde{R}\right) \stackrel{g}{\longleftarrow} \mathrm{Cl}\left(B^{\prime}\right)
$$

which are all isomorphisms by Theorem 1.1. and Corollary 3.5. Here it can be easily checked that $f\left(\operatorname{cl}\left(K_{B}\right)\right)$ and $g\left(\operatorname{cl}\left(K_{B^{\prime}}\right)\right)$ give the same element in $\mathrm{Cl}\left(B_{x}\right)$. (Use [7; Proposition (2.2.9)].)

Thus $\operatorname{cl}\left(K_{B}\right)=0$ if and only if $\operatorname{cl}\left(K_{B^{\prime}}\right)=0$ and this establishes the lemma.

Now let $k_{i}>0(i=1,2, \cdots, m)$ be as in the assumption of Theorem 1.2. (Recall that we always assume that $n_{i} \geqq k_{i}+k_{i+1}$ with $k_{0}=k_{m+1}$ $=0$.)

Then the Gorensteinness of $B_{R}\left(\begin{array}{c}n_{0}, n_{1}, \cdots, n_{m} \\ k_{1}, \cdots, k_{m}\end{array}\right)$ is equivalent to the Gorensteinness of $R$ and $R_{k(p)}\left(\begin{array}{c}n_{0}, n_{1}, \cdots, n_{m} \\ k_{1}, \cdots, k_{m}\end{array}\right)$ for all $p \in \operatorname{Spec}(R)$. Lemma 4.3 and Lemma 2.1 show that if $k_{s}>1$ then it is also equivalent to the Gorensteinness of $R$ and $B_{\tilde{k}(p)}\left(\begin{array}{c}n_{0}, \cdots, n_{s-1}-1, n_{s}-1, \cdots, n_{m} \\ k_{1}, \cdots, k_{s}-1, \cdots, k_{m}\end{array}\right)$ for any $\mathfrak{p} \in \operatorname{Spec}(R)$ where $\tilde{k}(\mathfrak{p}):=k(\mathfrak{p})\left[\left\{[i \mid j]_{s} \mid i=1\right.\right.$ or $\left.\left.j=1\right\}, 1 /[1 \mid 1]_{s}\right]$. Then by Lemma 4.2 (2) we see that it holds if and only if $R$ and

$$
B_{k(p)}\left(\begin{array}{c}
n_{0}, \cdots, n_{s-1}-1, n_{s}-1, \cdots, n_{m} \\
k_{1}, \cdots, k_{s}-1, k_{m}
\end{array}\right)
$$

are both Gorenstein. If we continue this process using Lemma 4.3 until 
each integer of the lower row of the parenthesis becomes 1 , then it will be concluded that it is equivalent to the Gorensteinness of $R$ and

$$
B_{k(\mathfrak{p})}\left(\begin{array}{c}
t_{0}+1, t_{1}+2, t_{2}+2, t_{m-1}+2, t_{m}+1 \\
1,1,1, \cdots, 1
\end{array}\right)
$$

for all $\mathfrak{p} \in \operatorname{Spec}(R)$. (Recall that $t_{i}=n_{i}-k_{i}-k_{i+1}$.)

Thus if we assume the validity of Proposition 4.1, then we shall get Theorem 1.2.

\section{$\S 5$. Final step of the proof}

Now we shall prove Proposition 4.1. Our main tool is the Poincaré series of graded rings which is defined as follows; If $A=\oplus_{n \geqq 0} A_{n}$ is an $N$-graded ring with Artinian $A_{0}$ and $A_{n}$ of finite length over $A_{0}$, then the Poincaré series $P_{A}(\lambda)$ of $A$ is the formal power series in $\lambda$ given by

$$
\sum_{n \geqq 0} \text { length }_{A_{0}}\left(A_{n}\right) \lambda^{n} \text {. }
$$

(We remark that this is always a rational function in $\lambda$ if $A$ is Noetherian.)

The reason why we are going to consider the Poincaré series is the following lemma due to R.P. Stanley.

Lemma 5.1 [11: Theorem 4.4]. Suppose that $A$ is a Cohen-Macaulay domain with $A_{0}$ a field and $d=\operatorname{dim} A . \quad$ Then $A$ is Gorenstein if and only if the equality;

$$
P_{A}(1 / \lambda)=(-1)^{d} \lambda^{a} P_{A}(\lambda)
$$

holds for some $a \in Z$.

First of all we shall compute the Poincaré series of $B_{R}\left(\begin{array}{c}r, s \\ 1\end{array}\right)$ where $r, s$ $\geqq 1$. (In the rest of this paper we assume that $R$ is a field.) If we denote it by $F_{r s}(\lambda)$ then it is given by

$$
F_{r s}(\lambda)=\sum_{n \geqq 0}\left(\begin{array}{c}
n+r-1 \\
r-1
\end{array}\right)\left(\begin{array}{c}
n+s-1 \\
s-1
\end{array}\right) \lambda^{n}
$$

where $\left(\begin{array}{l}u \\ 0\end{array}\right)=1$ for any $u \geqq 0$.

In fact each standard monomial of $B_{R}\left(\begin{array}{c}r, s \\ 1\end{array}\right)$ of degree $n$ is written as 


$$
\left[\begin{array}{c|c}
a_{1} & b_{1} \\
a_{2} & b_{2} \\
\vdots & \vdots \\
a_{n} & b_{n}
\end{array}\right]
$$

for some $1 \leqq a_{1} \leqq a_{2} \leqq \cdots \leqq a_{n} \leqq r$ and $1 \leqq b_{1} \leqq b_{2} \leqq \cdots \leqq b_{n} \leqq s$. (See [5; Theorem 12.1].) Thus the number of standard monomials of degree $n$ is given by $\left(\begin{array}{c}n+r-1 \\ r-1\end{array}\right)\left(\begin{array}{c}n+s-1 \\ s-1\end{array}\right)$. This establishes the equality (5.2).

On the other hand $F_{r s}(\lambda)$ should be written as a rational function in $\lambda$. In fact we get the equality;

$$
F_{r s}(\lambda)=\frac{f_{r s}(\lambda)}{(1-\lambda)^{r+s-1}}
$$

where

$$
f_{r s}(\lambda)=\sum_{j \geqq 0}\left(\begin{array}{c}
r-1 \\
j
\end{array}\right)\left(\begin{array}{c}
s-1 \\
j
\end{array}\right) \lambda^{j} \in Z[\lambda] .
$$

Remark that $\left(\begin{array}{l}u \\ v\end{array}\right)=0$ if $v<0$ or $v>u$.

To prove this equality we have only to notice that both functions in (5.2) and (5.3) are uniquely determined by the following condition.

$$
\begin{gathered}
F_{1 s}(\lambda)=\frac{1}{(1-\lambda)^{s}} \\
r F_{r+1, s}(\lambda)-s F_{r, s+1}(\lambda)=(r-s) F_{r s}(\lambda) .
\end{gathered}
$$

In the same way we define another formal power series $G_{r s}(\lambda)$ as follows:

$$
\text { If } r \geqq 1 \text { and } s \geqq 2 \text {, then }
$$

$$
G_{r s}(\lambda):=\sum_{n \geqq 1}\left(\begin{array}{c}
n+r-1 \\
r-1
\end{array}\right)\left(\begin{array}{c}
n+s-2 \\
s-1
\end{array}\right) \lambda^{n} \text {. }
$$

Then the same method as above shows that it is also written as a rational function in $\lambda$ :

$$
G_{r s}(\lambda)=\frac{g_{r s}(\lambda)}{(1-\lambda)^{r+s-1}}
$$

where

$$
g_{r s}(\lambda)=\sum_{j \geq 1}\left(\begin{array}{l}
r \\
j
\end{array}\right)\left(\begin{array}{l}
s-2 \\
j-1
\end{array}\right) \lambda^{j} \in Z[\lambda] .
$$

It is easy to check the following facts about those functions.

$$
\begin{aligned}
& \operatorname{deg} f_{r s}(\lambda)=\min \{r, s\}-1, \\
& \operatorname{deg} g_{r s}(\lambda)=\min \{r, s-1\} .
\end{aligned}
$$


(5.7) (i) If $r, s \geqq 1$, then $f_{r s}(\lambda)$ is a monic polynomial if and only if either $r=s$ or $(r-1)(s-1)=0$.

(ii) If $r \geqq 1$ and $s \geqq 2$, then $g_{r s}(\lambda)$ is monic if and only if either $r=s-1$ or $r=1$.

$$
\text { If } r \geqq 1 \text {, then }
$$

$$
\begin{aligned}
& F_{r r}(1 / \lambda)=-\lambda^{r} F_{r r}(\lambda), \\
& F_{r+1, r}(1 / \lambda)=\lambda^{r} G_{r, r+1}(\lambda), \\
& G_{r+1, r+1}(1 / \lambda)=-\lambda^{r} G_{r+1, r+1}(\lambda), \\
& G_{r, r+1}(1 / \lambda)=\lambda^{r} F_{r+1, r}(\lambda) .
\end{aligned}
$$

Remark 5.9. If $B_{R}\left(\begin{array}{c}r, s \\ 1\end{array}\right)$ is Gorenstein, then $F_{r s}(\lambda)$ satisfies the equality in Lemma 5.1. In particular the coefficient of highest degree term in $f_{r s}(\lambda)$ should be one. Thus in this case it must be $r=s$ or $(r-1)(s-1)$ $=0$. Conversely if $r=s$ or $(r-1)(s-1)=0$, then $F_{r s}(\lambda)$ certainly satisfies the condition in Lemma 5.1.

In such a way we can get the necessary and sufficient condition for $B_{R}\left(\begin{array}{c}r, s \\ 1\end{array}\right)$ to be Gorenstein.

We shall generalize this method to prove Proposition 4.1.

Now we denote the Poincare series of

$$
B_{R}\left(\begin{array}{c}
n_{0}, n_{1}, \cdots, n_{m} \\
1, \cdots, 1
\end{array}\right) \text { by } P_{n_{0}, n_{1}, \cdots n_{m}}(\lambda) .
$$

(Recall that $n_{0} \geqq 1, n_{m} \geqq 1$ and $n_{i} \geqq 2$ if $1 \leqq i<m$.)

Lemma 5.10. (1) $P_{n_{0}, n_{1}}(\lambda)=F_{n_{0}, n_{1}}(\lambda)$,

(2) $\quad P_{n_{0} n_{1} \cdots n_{m}}(\lambda)=F_{n_{0}, n_{1}-1}(\lambda) P_{n_{1}, \cdots, n_{m}}(\lambda)+G_{n_{0} n_{1}}(\lambda) P_{n_{1}-1, n_{2}, \ldots, n_{m}}(\lambda)$

whenever $m \geqq 2$.

Proof. We have already known (1). To prove (2) we must notice that each standard monomial of $B_{R}\left(\begin{array}{c}n_{0}, n_{1}, \cdots, n_{m} \\ 1, \cdots, 1\end{array}\right)$ is given by

$$
\left(\begin{array}{c|c|c|c|c|c}
a_{1}^{1} & b_{1}^{1} & b_{1}^{2} & & b_{1}^{m-1} & b_{1}^{m} \\
\vdots & \vdots & \vdots & \vdots & \vdots \\
a_{v_{1}}^{1} & \dot{b}_{v_{1}}^{1} & \dot{b}_{v_{2}}^{2} \\
\hline & a_{1}^{2} & a_{1}^{3} & & \dot{b}_{v_{m-1}}^{m-1} & \dot{b}_{v_{m}}^{m} \\
\hline & a_{1}^{m} & \\
\vdots & \vdots & & \\
& a_{v_{2}}^{2} & a_{v_{3}}^{3} & & a_{v_{m}}^{m} &
\end{array}\right)
$$


where $1 \leqq a_{1}^{i} \leqq a_{2}^{i} \leqq \cdots \leqq a_{v_{i}}^{i} \leqq n_{i-1}, 1 \leqq b_{1}^{i} \leqq b_{2}^{i} \leqq \cdots \leqq b_{v_{i}}^{i} \leqq n_{i}$ for any $i=1,2, \cdots, m$ and $a_{v_{i}}^{i}<n_{i-1}$ whenever $b_{v_{i}}^{i}=n_{i}$. (See (2.5).) Remark that the degree of this standard monomial is $v_{1}+v_{2}+\cdots+v_{m}$. Thus it is concluded that the number of standard monomials of degree $n$ in $B_{R}\left(\begin{array}{c}n_{0}, n_{1}, \cdots, n_{m} \\ 1, \cdots, 1\end{array}\right)$ is

$$
\begin{aligned}
& c_{n_{0} n_{1} \cdots n_{m}}^{(n)}=\sum_{v_{1}+v_{2}+\cdots+v_{m}=n}\left(\begin{array}{c}
v_{1}+n_{0}-1 \\
n_{0}-1
\end{array}\right)\left\{\left(\begin{array}{c}
v_{1}+n_{1}-2 \\
n_{1}-2
\end{array}\right)\left(\begin{array}{c}
v_{2}+n_{1}-1 \\
n_{1}-1
\end{array}\right)\right. \\
& \left.+\left(\begin{array}{c}
v_{1}+n_{1}-2 \\
n_{1}-1
\end{array}\right)\left(\begin{array}{c}
v_{2}+n_{1}-2 \\
n_{1}-2
\end{array}\right)\right\} \cdots\left\{\left(\begin{array}{c}
v_{m-1}+n_{m-1}-2 \\
n_{m-1}-2
\end{array}\right)\left(\begin{array}{c}
v_{m}+n_{m-1}-1 \\
n_{m}-1
\end{array}\right)\right. \\
& \left.+\left(\begin{array}{c}
v_{m-1}+n_{m-1}-2 \\
n_{m-1}-1
\end{array}\right)\left(\begin{array}{c}
v_{m}+n_{m-1}-2 \\
n_{m-1}-2
\end{array}\right)\right\}\left(\begin{array}{c}
v_{m}+n_{m}-1 \\
n_{m}-1
\end{array}\right) .
\end{aligned}
$$

In particular we have that

$$
\begin{aligned}
c_{n_{0} n_{1} \cdots n_{m}}^{(n)}= & \sum_{v_{1}=0}^{n}\left\{\left(\begin{array}{c}
v_{1}+n_{0}-1 \\
n_{0}-1
\end{array}\right)\left(\begin{array}{c}
v_{1}+n_{1}-2 \\
n_{1}-2
\end{array}\right) c_{n_{1} n_{2} \cdots n_{m}}^{\left(n-v_{1}\right)}\right. \\
& \left.+\left(\begin{array}{c}
v_{1}+n_{0}-1 \\
n_{0}-1
\end{array}\right)\left(\begin{array}{c}
v_{1}+n_{1}-2 \\
n_{1}-1
\end{array}\right) c_{n_{1}-1, n_{2}, \cdots, n_{m}}^{\left(n-v_{0}\right)}\right\}
\end{aligned}
$$

Since $P_{n_{0} n_{1} \cdots n_{m}}(\lambda)=\sum_{n_{0} \geq 0} c_{n_{0} n_{1} \cdots n_{m}}^{(n)} \lambda^{n}$, this establishes the lemma.

As the corollary of this lemma we can write $P_{n_{0} n_{1} \cdots n_{m}}(\lambda)$ as a rational function in $\lambda$.

Corollary 5.11 .

$$
P_{n_{0} n_{1} \cdots n_{m}}(\lambda)=\frac{p_{n_{0} n_{1} \cdots n_{m}}(\lambda)}{(1-\lambda)^{d}}
$$

where $p_{n_{0} n_{1}}(\lambda)=f_{n_{0} n_{1}}(\lambda)$,

$$
p_{n_{0} n_{1} \cdots n_{m}}(\lambda)=f_{n_{0} n_{1}-1}(\lambda) p_{n_{1} n_{2} \cdots n_{m}}(\lambda)+g_{n_{0} n_{1}}(\lambda) p_{n_{1}-1, n_{2}, \cdots, n_{m}}(\lambda) \in Z[\lambda] \quad \text { if } m \geqq 2
$$

and

$$
d=n_{0}+n_{m}+2 \sum_{i=1}^{m-1} n_{i}-2 m+1
$$

(Remark that the integer $d$ in this lemma is the Krull dimension of the ring $B_{R}\left(\begin{array}{c}n_{0}, n_{1}, \cdots, n_{m} \\ k_{1}, \cdots, k_{m}\end{array}\right)$. See [3; Lemma 2.3]. And also remark that all the coefficients of the polynomial $p_{n_{0} n_{1} \cdots n_{m}}(\lambda)$ are non-negative integers.)

Here by using (5.6) we know inductively that 


$$
\begin{aligned}
& \operatorname{deg} f_{n_{0} n_{1}-1} p_{n_{1} n_{2}}=\min \left\{n_{0}, n_{1}-1\right\}+\min \left\{n_{1}, n_{2}\right\}-2, \\
& \operatorname{deg} f_{n_{0} n_{1}-1} p_{n_{1} n_{2} \cdots n_{m}}=\min \left\{n_{0}, n_{1}-1\right\}+\min \left\{n_{1}, n_{2}-1\right\} \\
& +\min \left\{n_{m-1}-1, n_{m}\right\}+\sum_{i=2}^{m-2} \min \left\{n_{i}, n_{i+1}\right\}-m+1 \\
& \text { if } m \geqq 3 \text {, } \\
& \operatorname{deg} p_{n_{0} n_{1} \cdots n_{m}}=\operatorname{deg} g_{n_{0} n_{1}} p_{n_{1-1}, n_{2}, \cdots, n_{m}} \\
& =\min \left\{n_{0}, n_{1}-1\right\}+\min \left\{n_{m-1}-1, n_{m}\right\} \\
& +\sum_{i=1}^{m-2} \min \left\{n_{i}, n_{i+1}\right\}-m+1 \quad \text { if } m \geqq 2 \text {. }
\end{aligned}
$$

In particular we have the following lemma.

LEMMA 5.12. $\operatorname{deg} f_{n_{0} n_{1}-1} p_{n_{1} \cdots n_{m}} \leqq \operatorname{deg} g_{n_{0} n_{1}} p_{n_{1}-1, n_{2} \cdots n_{m}}$ and the equality holds if and only if $n_{1}<n_{2}$.

Now let us prove Proposition 4.1. If $m=1$, then we have already proved it in Remark 5.9. So in the following we assume that $m \geqq 2$.

Suppose that $B_{R}\left(\begin{array}{c}n_{0}, n_{1}, \cdots, n_{m} \\ 1, \cdots, 1\end{array}\right)$ is a Gorenstein ring. Then $P_{n_{0} n_{1} \cdots n_{m}}(\lambda)$ satisfies the condition in Lemma 5.1 , so in particular the polynomial $p_{n_{0} n_{1} \cdots n_{m}}(\lambda)$ is monic. Thus Lemma 5.12 gives that $n_{1} \geqq n_{2}$ and $g_{n_{0} n_{1}} p_{n_{1}-1, n_{2} \cdots n_{m}}$ is also monic. By (5.7) this is equivalent to say that $p_{n_{1}-1, n_{2} \cdots n_{m}}$ is monic and either $n_{0}+1=n_{1} \geqq n_{2}$ or $n_{0}=1, n_{1} \geqq n_{2}$. If we continue this process, we can easily get one of the four conditions in Proposition 4.1.

Conversely assume that $n_{0}+1=n_{1}=\cdots=n_{m-1}=n_{m}+1$. For the convenience let us denote $r=n_{0}=n_{m}$ and

$$
\begin{aligned}
S_{m}(\lambda) & :=\underbrace{P_{r, r+1, \ldots, r+1, r}(\lambda)}_{m+1} \\
T_{m}(\lambda) & :=\underbrace{P_{r+1, r+1, \ldots, r+1, r}}_{m+1}(\lambda)
\end{aligned}
$$

with $S_{1}=F_{r r}$ and $T_{1}=F_{r+1, r}$.

Then by Lemma 5.10 we see that

$$
\begin{aligned}
& {\left[\begin{array}{l}
S_{m}(\lambda) \\
T_{m}(\lambda)
\end{array}\right]=\left[\begin{array}{c}
G_{r, r+1}(\lambda), F_{r r}(\lambda) \\
G_{r+1, r+1}(\lambda), F_{r+1, r}(\lambda)
\end{array}\right]\left[\begin{array}{c}
S_{m-1}(\lambda) \\
T_{m-1}(\lambda)
\end{array}\right]} \\
& =\cdots=\left[\begin{array}{c}
G_{r, r+1}(\lambda), F_{r r}(\lambda) \\
G_{r+1, r+1}(\lambda), F_{r+1, r}(\lambda)
\end{array}\right]^{m}\left[\begin{array}{l}
0 \\
1
\end{array}\right]
\end{aligned}
$$

Thus by (5.8) we have an equality;

$$
\left[\begin{array}{l}
S_{m}(1 / \lambda) \\
T_{m}(1 / \lambda)
\end{array}\right]=\lambda^{m r}\left[\begin{array}{c}
F_{r+1, r}(\lambda),-F_{r r}(\lambda) \\
-G_{r+1, r+1}(\lambda), G_{r, r+1}(\lambda)
\end{array}\right]^{m}\left[\begin{array}{l}
0 \\
1
\end{array}\right]
$$


Comparing these equalities we easily conclude that

$$
S_{m}(1 / \lambda)=-\lambda^{m r} S_{m}(\lambda)
$$

Hence $B_{R}\left(\begin{array}{c}r, r+1, \cdots, r+1, r \\ 1, \cdots \cdots, 1\end{array}\right)$ is Gorenstein by virtue of Lemma 5.1.

Next we assume that $n_{0}=1$ and $n_{1}=n_{2}=\cdots=n_{m-1}=n_{m}+1(=: r)$. If $r=2$, this case is included in the previous one, and there is nothing to say. So we may take $r>2$. Then using Lemma 4.3 (ii), we have that $B_{R}\left(\begin{array}{c}1, r, \cdots, r, r-1 \\ 1, \cdots, 1\end{array}\right)$ is Gorenstein if and only if $B_{R}\left(\begin{array}{c}r-1, r, \cdots, r, r-1 \\ \underbrace{1, \cdots, 1}_{m-1}\end{array}\right)$ is Gorenstein. Thus by the previous case it is concluded that $B_{R}\left(\begin{array}{c}1, r, \cdots, r, r-1 \\ 1, \cdots \cdots, 1\end{array}\right)$ is always Gorenstein whenever $r \geqq 2$.

The other cases in Proposition 4.1 can be treated as in the same method using Lemma 4.3, and so we omit the detail.

\section{REFERENCES}

[1] N. Bourbaki, Algèbre Commutative, Hermann, Paris, 1965.

[2] W. Bruns, Die Divisorenklassengruppen der Restklassenringe von Polynomringen nach Determinantenidealen, Rev. Roumaine Math. Pures Appl., XX, No. 10 (1975), 1109-1111.

[ 3 ] C. DeConcini and E. Strickland, On the variety of complexes, Adv. in Math., 41 (1981), 57-77.

[4] D. Eisenbud, Introduction to algebras with straightening laws. Ring theory and algebra III, proceeding of the third Oklahoma conference, Ed. B. McDonald, Marcel Dekker, New York, 1980.

[ 5 ] C. DeConcini, D. Eisenbud and C. Procesi, Hodge algebras, preprint.

[6] J. Eagon and M. Hochster, Cohen-Macaulay rings, invariant theory, and the generic perfection of determinantal loci, Amer. J. Math., 43 (1971), 1020-1058.

[ 7 ] S. Goto and K. Watanabe, On graded rings, I, J. Math. Soc. Japan, 30 (1978), 172-213.

[8] J. Herzog and E. Kunz, Der kanonische Modul eines Cohen-Macaulay Rings, Lecture Note in Math., 238, Springer (1971).

[ 9 ] T. Svanes, Coherent cohomology on Schubert subschemes of flag schemes and applications, Adv. in Math., 14 (1974), 369-453.

[10] K. Watanabe et al., On tensor products Gorenstein rings, J. Math. Kyoto Univ., 9-3 (1969), 413-423.

[11] R. Stanley, Hilbert functions of graded algebras, Adv. in Math., 28 (1978), 57-83.

Department of Mathematics

Nagoya University

Chikusa-ku, Nagoya 464

Japan 